\title{
FIRST REPORTED USE OF ZIDOVUDINE FOR PREVENTION OF PERINATAL HIV TRANSMISSION IN A PREMATURE CHILD ON ECMO
}

M. Emonts ${ }^{1,2}$, H. Waalewijn ${ }^{3}$, A. Colbers ${ }^{3}$, A. Pickering ${ }^{1}$, D. Burger ${ }^{3}$

${ }^{1}$ Great North Children's Hospital- Newcastle upon Tyne Hospitals NHS Foundation Trust, Paediatric Immunology- Infectious Diseases and Allergy, Newcastle upon Tyne, United Kingdom. ${ }^{2}$ Newcastle University, Institute of Cellular Medicine, Newcastle upon Tyne, United Kingdom. ${ }^{3}$ Radboud Institute for Health Sciences- Radboud university medical centre, Department of Pharmacy, Nijmegen, The Netherlands.

\section{Background}

The effects of extra corporal membrane oxygenation (ECMO) on pharmacokinetics (PK) of drugs is difficult to predict. Generally, an increased volume of distribution, decreased drug elimination, and sequestration of the drug to the ECMO circuit are factors potentially influencing the PK of drugs during ECMO. Zidovudine is the only antiretroviral agent suitable for intravenous use in newborns for the prevention of perinatal HIV transmission. No reported cases of the influence of ECMO on the PK of zidovudine in prematures were found.

\section{Case Presentation Summary}

A premature child (32 weeks) born from a virologically suppressed HIV infected mother required ECMO to undergo the resection of an intrathoracic lesion compromising the lungs. IV zidovudine was administered for the prevention of HIV transmission. To cope with the anticipated increased volume of distribution $\left(\mathrm{V}_{d}\right)$ and in order to avoid the risks caused by under treatment, IV zidovudine was dosed $9 \mathrm{mg} / \mathrm{kg} /$ day $(150 \%$ of the dose normally used in premature infants) for the duration of ECMO. Plasma samples were taken before, during and after ECMO. Samples were analysed using LC-MS technology. Therapeutic drug monitoring (TDM) was used to observe treatment and PK parameters were calculated using non-compartmental analysis in WinNonlin. Parents consented to the presentation of these data.

With clearance $0.62 \mathrm{~L} / \mathrm{h}, \mathrm{Vd}_{\mathrm{d}} 3.3 \mathrm{~L}$, and $\mathrm{t} 1 / 2$ of $3.6 \mathrm{~h}$, zidovudine concentrations remained above $0.8 \mathrm{mg} / \mathrm{L}$ during ECMO. While pharmacokinetic reports on prematures are highly variable with no Ctroughs or AUCs reported, this exposure has been correlated to increased safety risks in earlier studies.[1] No adverse events were reported in this case and zidovudine levels returned to normal on standard doses after ECMO cessation.[2,3] The HIV proviral DNA PCR in the child was negative at 3 months of age.

Learning Points/Discussion

In future occasions, standard IV dosing of zidovudine in premature children on ECMO is recommended under the guidance of TDM.

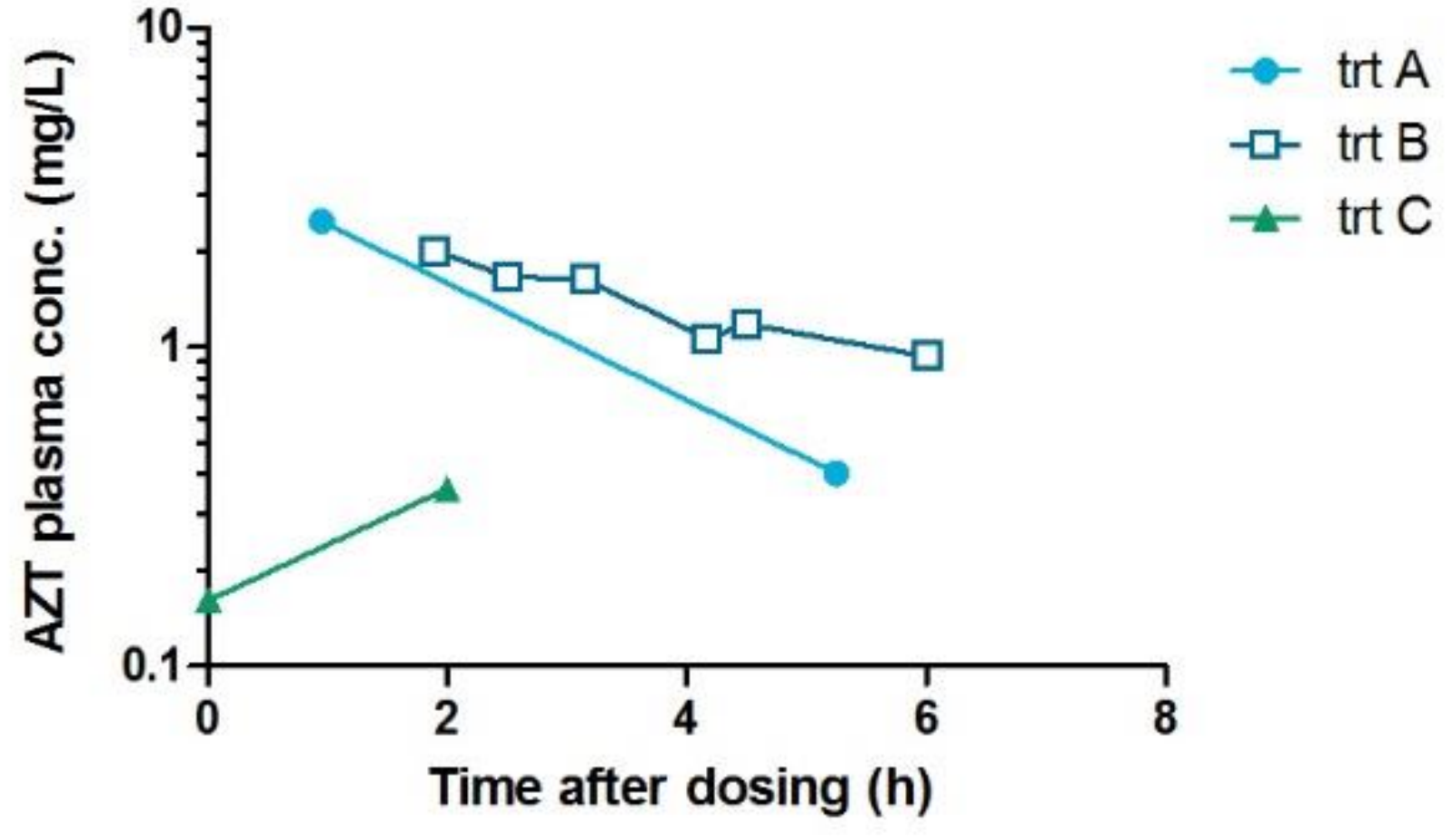

Figure 1. Zidovudine levels post dosing

- A. Zidovudine levels with iv medication, no ECMO

$\square$ B. Zidovudine levels with iv medication on ECMO

$\triangle$ C. Zidovudine levels with oral medication

\section{References}

[1] Balis, F.M., et al. Ann Intern Med, 1989. 110(4): p. 27985.

[2] Mirochnick, M., E. Capparelli, and J. Connor. Clin Pharmacol Ther, 1999. 66(1): p. 16-24.

[3] Pacifici, G.M. Early Hum Dev, 2005. 81(9): p. 773-80.

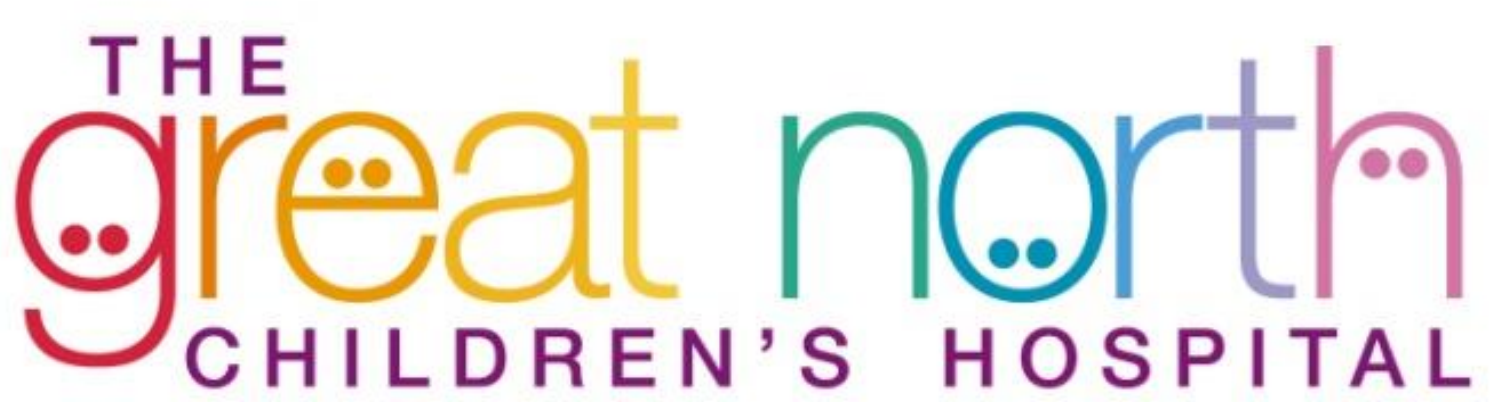

